\title{
Avaliação em promoção da saúde: foco no "município saudável"
}

\section{Health promotion evaluation: focus on "healthy cities"}

\section{Marco Akermana, Rosilda Mendes ${ }^{\text {b }}$ Cláudia Maria Bógusc, Márcia Faria Westphald, Aluisio Bichire e Mylene Lyra Pedroso $^{f}$}

aFaculdade de Medicina do ABC. Santo André, SP, Brasil. 'becretaria da Saúde. Prefeitura Municipal de São Paulo. São Paulo, SP, Brasil. Instituto de Saúde da Secretaria de Estado da Saúde de São Paulo. São Paulo, SP, Brasil. dD epartamento de Prática de Saúde Pública da Faculdade de Saúde Pública da U niversidade de São Paulo. São Paulo, SP, Brasil. eSecretaria da Saúde do M unicípio de Bertioga. Bertioga, SP, Brasil. fSecretaria Municipal de Meio Ambiente do Município de Bertioga. Bertioga, SP, Brasil

\section{Descritores}

Cidade saudável. Promoção da saúde. Avaliação. Participação social.

\section{Keywords}

Healthy City. Health promotion. Evaluation. Social participation.

\section{Resumo}

Sendo o movimento de cidades/municípios saudáveis uma estratégia de promoção da saúde, elaborou-se um trabalho de atualização de informações com o objetivo de contextualizar o debate da avaliação no campo da promoção da saúde, apontando os princípios que devem nortear o estabelecimento de um processo avaliativo e problematizar este tema quanto aos projetos de cidades/municípios saudáveis. Propõese uma tipologia que classifica os artigos indexados nas bases de dados MedLine e Lilacs, entre 1985 e 2000, sobre o tema "cidades/municípios saudáveis" de acordo com a ênfase de cada artigo analisado. Os artigos que enfatizam iniciativas de avaliação de projetos são analisados com maior detalhamento, tabulando-os em função da metodologia utilizada, dos instrumentos aplicados, dos indicadores produzidos, dos resultados obtidos e da análise crítica do modelo adotado. Destacam-se iniciativas de avaliação de "cidades/municípios saudáveis", que se aproximariam mais dos princípios da "promoção da saúde", e que adotam a avaliação, mas como instrumento de construção de capacidades e fortalecimento de grupos populacionais envolvidos com projetos nessa temática.

\footnotetext{
Abstract

Since the Healthy City movement is a health promotion strategy, an update study was carried out to put into context the health promotion evaluation debate stressing the principles to be considered in an evaluation initiative and in the problematic of Health Cities projects. A literature review of "Healthy Cities" was conducted. A typology based on of Healthy Cities papers found in MEDLINE, LILACS and published in the "Health Promotion International" between 1985 and 2000 was proposed taking into consideration the main focus of each paper. Articles on evaluation were analyzed in more details according to stressing methods, research tools, indicators, study results, and critical appraisal of their models. Finally, it is highlighted initiatives that would come close to the principles of "Health Promotion" and adopt evaluation as a tool for building up capabilities and the empowerment of community groups involved with Healthy Cities initiatives.
}

Correspondência para/Correspondence to: Marcos Akerman Rua Dona Brígida, 232 casa 32 04111-080 São Paulo, SP, Brasil Fone / Fax: 55731930

Email: akermanm.ops@terra.com.br 


\section{INTRODUÇÃO}

O objetivo do presente trabalho é atualizar informações sobre cidades/municípios saudáveis contextualizando o debate da avaliação no campo da Promoção da Saúde, em geral, e problematizar o tema no que concerne aos projetos de cidades/municípios saudáveis.*

A primeira definição de Cidades Saudáveis foi elaborada por Hancock ${ }^{23}$ e Duhl, ${ }^{10} \mathrm{em}$ 1986. Os autores ressaltam a importância histórica do processo de tomada de decisão dos governos locais no estabelecimento de condições para a saúde, para interferir nos determinantes sociais, econômicos e ambientais, por meio de estratégias como planejamento urbano, "empowerment"** comunitário e participação da população. Segundo a concepção dos autores, uma "cidade saudável" é aquela que está continuamente criando e melhorando o ambiente físico e social, fortalecendo os recursos comunitários que possibilitam às pessoas se apoiarem mutuamente no sentido de desenvolverem seu potencial e melhorarem sua qualidade de vida.

Mendes ${ }^{36}$ foi um dos primeiros autores brasileiros a abordar o tema. Considera o projeto Cidades/Municípios Saudáveis como um "projeto estruturante do campo da saúde", em que os atores sociais (governo, organizações da sociedade civil, organizações não governamentais) procuram, por meio da "gestão social", transformar a cidade em um espaço de "produção social da saúde”. Desta forma, a saúde é entendida como qualidade de vida e é considerada objeto de todas as políticas públicas, dentre estas, as políticas de saúde.

Elaborar uma metodologia que permita avaliar o processo de desenvolvimento dos projetos Cidades/ Municípios Saudáveis implica reconhecer diversos fatores e acordar múltiplas concepções, tais como: ${ }^{37}$

- ser congruente com os princípios de desenvolvimento humano e social sustentável;

- ser participativo para fortalecer a capacidade da população e das organizações;

- ser formativo e;

- adotar métodos mistos, quantitativos e qualitativos.

Sugere-se que as iniciativas implementadas sejam avaliadas levando-se em conta quatro aspectos dos projetos: políticas públicas, participação social, intersetorialidade e sustentabilidade. ${ }^{41,42}$

Já há alguns anos, tem-se colocado a necessidade de avaliar programas e atividades de promoção da saúde. Documentos e artigos recentes têm avaliado as evidências em relação à efetividade de programas fazendo recomendações para formuladores de política. ${ }^{26,33,40,61}$

Esta é uma questão complexa, uma vez que o marco conceitual e metodológico da avaliação deve reconhecer a saúde como qualidade de vida e, portanto, a complexidade de seu enfoque de atuação sobre os determinantes sociais de saúde. Em consequiência, a avaliação de cidades/municípios saudáveis deve refletir os aspectos fundamentais da promoção da saúde, tais como alcançar a eqüidade, a participação social e a intersetorialidade para obtenção de melhor qualidade de vida. ${ }^{42} \mathrm{~A}$ pesquisa desenvolvida por Goumans,,$^{20}$ sobre cidades saudáveis na Holanda e Inglaterra, discute a dificuldade de avaliação, dada a impossibilidade de variáveis serem isoladas no contexto dos projetos. Por isso, não é fácil produzir evidências sobre a contribuição específica das cidades saudáveis.

\section{DEBATE DA AVALIAÇÃO NO CAMPO DA PROMOÇÃO DA SAÚDE}

A cobrança crescente da sociedade civil por resultados visíveis que atestem o correto uso dos recursos públicos e melhorem sua qualidade de vida; o limitado sucesso de muitos programas; o desconhecimento dos efeitos e impactos produzidos pelos investimentos realizados nas políticas públicas e nos programas e projetos de desenvolvimento social têm feito com que os governos, organizações não governamentais, fundações de corporações públicas e privadas, principalmente agências internacionais de fomento e os países doadores reivindiquem cada vez mais a inclusão de metodologias de monitoramento e avaliação de processo, resultados e impacto dos programas implementados. Na América Latina, são raros os programas que aprofundam seus esforços avaliativos, no sentido de mostrar efeitos e impactos. A maior parte deles restringe-se apenas à descrição das discrepâncias entre o realizado e o programado, e entre os re-

*No Brasil, a discussão de denominação desses projetos caminha nessa mesma direção. 0 termo foi debatido no "I Fórum Brasileiro de M unicípios Saudáveis" ocorrido em Sobral, em 1998. A denominação "Cidade Saudável" foi defendida porque "cidade" estaria relacionada a polis, local onde as pessoas assumem sua condição de cidadão, e pode, além disso, melhor representar a intensa urbanização que o país vive e os novos problemas de saúde que advém do atual estágio de desenvolvimento dos aglomerados humanos, caracterizados na forma de cidades, da vida urbana. Já o termo "M unicípio Saudável" foi defendido por refletir a organização de grande parte dos aglomerados no país, que contém núcleos habitacionais estruturados economicamente na produção rural localizados em torno de um núcleo principal de características urbanas mais evidentes. 0 que se observa é que ambas as denominações são utilizadas, e isso tem relação com o período histórico de aproximação das cidades ao ideário. As cidades que foram sensibilizadas pelo governo canadense a adotar políticas nesta perspectiva utilizam o termo "Cidades Saudáveis", enquanto outras, incentivadas por outros órgãos, como a O rganização PanAmericana de Saúde, referem-se ao "Município Saudável". Desta forma consideram-se as designações “Cidades" e "Municípios Saudáveis" como sinônimos.

**O movimento de promoção da saúde tem tomado como um dos conceitos prioritários o de empowerment, que traz na sua raiz o significado de poder, ou ganho de poder para tomar decisões, realizar ações, individual e coletivamente, visando à eficácia política, melhoria da qualidade de vida e justiça social. (Wallerstein, ${ }^{57}$ 1992). 
cursos implementados e as coberturas alcançadas. ${ }^{7}$

O campo da promoção da saúde não está imune a essas demandas sociais. ${ }^{50}$ Uma vez que requer múltiplos enfoques, depende de informações advindas de campos disciplinares distintos e demanda desenvolvimento de longo prazo para produzir resultados, fica inadequado avaliar sua prática a partir dos métodos usualmente utilizados para avaliar o cuidado médico e a prevenção de doenças. ${ }^{40}$

Por isso, o Relatório Health Promotion Evaluation: Recommendations to Policymakers publicado em 1998 pelo Escritório Regional para a Europa da Organização Mundial da Saúde (OMS) ${ }^{61}$ entende que a implementação do conceito de promoção da saúde da Carta de Ottawa ${ }^{43}$ - o processo de capacitar pessoas e populações para aumentar o seu controle para melhorar a sua saúde - depende de ações que sejam empowering, participativas, holísticas, intersetoriais, equânimes, sustentáveis e multi-estratégicas. Há que se desenvolver estratégias avaliativas que ultrapassem as fronteiras da tradicional prática em saúde, centrada no biológico e na prevenção de doenças através da redução de riscos individuais.

Baseado nesses princípios, o grupo de trabalho, que elaborou o citado Relatório, estabeleceu que para uma avaliação de iniciativas de promoção da saúde ser apropriada ela deve, imprescindivelmente, conter os seguintes aspectos:

- Participação: deve envolver, de uma maneira apropriada, em cada estágio da avaliação, todos aqueles que têm interesse legítimo na iniciativa que está sendo avaliada.

- Múltiplos métodos: deve ser delineado utilizando-se elementos de vários campos disciplinares, lançando mão de vários procedimentos para coletar dados, assegurando que seja utilizado um mix de informações relacionadas ao processo e resultado.

- Capacitação (capacity building): deve aprimorar a capacidade de indivíduos, organizações e governos de equacionar relevantes problemas de promoção da saúde.

- Adequabilidade (appropriateness): deve ser planejada levando-se em conta a natureza complexa da intervenção e o seu impacto de longo prazo.

\section{PAN O RAMA DA LITERATU RA}

Utilizando-se as palavras-chaves "cidade" (city) "saudável" (healthy) foi realizada uma revisão de artigos publicados, entre 1985 e 2000, indexados nas bases de dados especializadas na área de saúde, Medline e Lilacs. O Health Promotion International
- importante periódico internacional de promoção da saúde, indexado no Medline, teve seus exemplares, do mesmo período, revisados manualmente para eventual inclusão de artigos que por ventura não tivessem sido recuperados pelo Medline Os estudos obtidos foram analisados em função de uma tipologia que os classificou em:

- "Texto Geral": aborda o tema cidade/município saudável de uma forma ampla, levando em conta aspectos históricos e conceituais, princípios, diretrizes, como estratégia de promoção da saúde, e outros;

- "Estudo de Caso": focaliza relato de experiências de implementação de projetos específicos, sem apresentar estratégias e resultados avaliativos;

- "Estudo Avaliativo": focaliza relato de experiências de implementação de projetos específicos, com apresentação de estratégias e resultados avaliativos;

- "Outros": estudos que não se enquadram em nenhuma das alternativas anteriores.

Os "Estudos Avaliativos" são analisados com maior detalhamento, tabulando-os em função da metodologia utilizada, dos instrumentos aplicados, dos indicadores produzidos, dos resultados obtidos e da análise crítica do modelo adotado.

Werna et al $^{59}$ publicaram, em 1998, um livro abrangente sobre a implantação de iniciativas por cidades saudáveis em países em desenvolvimento. Os autores construíram sua reflexão a partir de 108 referências bibliográficas sendo que, apenas 11 versam sobre a temática da avaliação de projetos de $c i$ dades saudáveis. Destes, oito são relatórios de consultoria para agências internacionais e, portanto, de circulação restrita. Isso é um indicativo de que a temática da avaliação ainda é pouco explorada pelos estudiosos do tema.

Dos 43 artigos encontrados nas bases Medline, Lilacs e na revista Health Promotion International, abordando o tema cidades saudáveis, apenas, seis versam, especificamente, sobre o tema da avaliação, sendo que dois abordam apenas princípios e caminhos metodológicos, sem se ocupar de nenhum esforço avaliativo concreto.

Vinte e dois artigos localizados no Medline não foram publicados no Health Promotion International. A maioria (14) são "Textos Gerais" $8,14,16,23,24,27,30,32,34,38,39,45,49,51,55$ abordando a história do movimento, as direções que a prática da promoção da saúde pode assumir após o advento de cidades saudáveis, conceitos e princípios do movi- 
mento, enquanto oito artigos 4,10,15,17,19,25,52-54 são “Estudos de Casos" que descrevem a implementação de projetos específicos, sem se aprofundar na análise de efeitos ou impactos como um todo.

Destes "Estudos de Caso", seis estão relacionados com projetos de redução de fatores de risco ou aperfeiçoamento da qualidade de serviços de saú$\mathrm{de}^{10,15,19,25,53,54}$ como parte de projetos de cidades saudáveis. Entretanto, não transparecem ser projetos abrangentes que levem em conta a intersetorialidade, a participação e o desenvolvimento social.

Para tais estudos, são apresentados resultados específicos, o que denota algum grau de esforço avaliativo. Gonzales ${ }^{53}$ mostra maiores níveis de prevenção em relação ao álcool, ao cigarro e sedentarismo para os grupos mais ricos das cidades em comparação com os mais pobres, e Howell \& Doorley $^{25}$ analisam a atitude de um grupo de empregados de uma empresa frente ao tabagismo passivo.

Quanto à avaliação de resultados no que concerne à melhoria da qualidade de serviços de saúde em projetos de cidades saudáveis, Stephenson et al ${ }^{10}$ analisam a satisfação de 47 mulheres com os serviços de pré-natal em São Petersburgo, na Rússia, enquanto na mesma "São Petersburgo Saudável", onde se decidiu que a maior prioridade do projeto seria a redução da mortalidade materna, houve declínio nas taxas de mortalidade materna e aborto e aumento no uso de meios contraceptivos. Mas, apesar da especificidade dos indicadores, os autores afirmam que tal sucesso deveu-se ao "amplo envolvimento da sociedade".

Há ainda artigos que lançam mão de uma rede de cidades saudáveis, para estudos mais específicos, como o de Garcia ${ }^{19}$ que analisa aspectos educacionais em escolas de medicina das cidades que compõem a rede sobre a ressuscitação cárdio-pulmonar, concluindo que há diferenças importantes quanto às horas de treinamento dedicadas a esse procedimento.

Os artigos obtidos na base Lilacs foram oito. ${ }^{1,2,9,13,28,31,48,60}$ Diferentemente da literatura internacional, que apresenta artigos sobre cidades saudáveis, indexados na base Medline a partir de 1989, o primeiro texto brasileiro, indexado, foi escrito em 1993. Dos oito artigos obtidos, seis recaem na categoria "texto geral", que discute a pertinência do movimento para o Brasil; ${ }^{13}$ apresenta os principais conceitos de forma analítica ${ }^{9,60}$ e entende o movimento de cidades saudáveis como estratégias de gestão municipal, ${ }^{31}$ de planejamento ${ }^{28}$ e de compromisso político. ${ }^{2}$ Os dois "estudos de caso" relatam a implementação de um projeto intersetorial em Fortale- $\mathrm{za}^{48}$ e do "Observatório de Qualidade de Vida" em Campinas, ${ }^{1}$ também uma intervenção de cunho intersetorial.

Nenhum dos artigos indexados na base Lilacs aborda a temática da avaliação de forma abrangente, ou aplica metodologias de avaliação de resultados ou impacto a algum projeto específico de cidadel município saudável. Isso poderia ser explicado, talvez, pelo pouco tempo de maturação dos projetos no Brasil, que têm na cidade de São Paulo a sua iniciativa pioneira datada de 1991, mas interrompida desde $1993 .{ }^{37}$

Treze artigos foram publicados naHealth Promotion International. ${ }^{5,6,18,20,21,29,35,39,44,47,48,56}$ Esses artigos apresentam maior grau de especificidade que a seleção anterior, principalmente, no que concerne a "estudos avaliativos" de projetos específicos. Dos 13 artigos selecionados, quatro são "texto geral" $3,29,35,44,56$ que vêem o movimento de cidades/municípios saudáveis como expressão da "nova saúde pública", tecendo considerações sobre projetos em países desenvolvidos; três recaem na categoria "estudo de caso"18,21,22 e examinam o conjunto de projetos e redes de cidades na Europa e Canadá, do ponto de vista de sua implementação e implicações para a ciência política e administrativa; e seis abordam a questão da avaliação sendo que quatro artigos apresentam estudos avaliativos de projetos específicos ${ }^{5,6,39,44}$ e dois classificados como "outro" 46 discutem princípios e critérios para a avaliação de comunidades saudáveis.

\section{AVALIAÇÃO : ABORDANDO PRIN CÍPIOSE MÉTO DOS}

Além de levantar princípios e fazer recomendações para avaliação de iniciativas de promoção da saúde, ${ }^{46}$ o Relatório Health Promotion Evaluation: Recommendations to Policymakers, publicado em 1998 pelo Escritório Regional para a Europa da $\mathrm{OMS}^{61}$ faz o mesmo exercício para healthy communities initiatives, propugnando a adoção de três princípios e seis focos de análise de conteúdo dos projetos que "podem guiar o desenvolvimento de conhecimento e avaliação em, de, e para iniciativas de comunidades saudáveis". Entretanto, Poland ${ }^{46,47}$ adverte que esta não é uma lista definitiva e que, cada grupo local necessitará considerar qual desses elementos melhor se aplica à sua realidade e se há outros aspectos a serem adicionados.

Como princípios a serem adotados numa proposta de avaliação de "comunidades saudáveis", Poland, 46,47 destaca:

- a necessidade de uma abordagem participativa 
integrada ao programa que leve em conta valores, utilize múltiplos métodos, enfoque os processos, e não apenas os resultados, e que dê feedback oportuno e compreensível aos participantes;

- a incorporação de uma ciência social com perspectiva crítica que equacione aspectos de poder, que explicite as bases da teoria adotada, posições políticas e ideológicas, paradoxos entre teoria e prática, a dialética entre estrutura social e experiência individual e que sensibilize os participantes para o grau de incerteza dos fenômenos sociais;

- o desenvolvimento de um modelo conceitual, delineado localmente, ${ }_{2}$ que seja consistente com os propósitos e estratégias do movimento sensível às necessidades e perspectivas dos vários atores sociais e permeável ao uso de múltiplos métodos de abordagem.

Poland ${ }^{46,47}$ alerta, também, que apesar de advogar firmemente uma avaliação participativa, não deixa de prestar atenção cuidadosa aos aspectos políticos e epistemológicos da teoria e dos métodos adotados. Para isso, o autor aponta as seguintes referências que seriam as bases para a produção do conhecimento e para o desenvolvimento de metodologias de avaliação:

- construção da visão do que é uma comunidade saudável, analisando se a comunidade está aproximando-se ou afastando-se dessa visão;

- princípios, conceitos e estratégias do movimento de comunidades saudáveis - equidade, participação social, ação multi-setorial, desenvolvimento sustentável, determinação social da saúde, desenvolvimento social, liderança com responsabilidade e capacidade de gerar recursos (stewardship) - como o ponto de partida lógico para avaliação;

- barreiras e facilitadores do crescimento e realização do projeto, avaliando o que melhor pode predizer sucesso ou insucesso da experiência examinada;

- setor e unidade de análise dirigindo o exame avaliativo para as conexões intersetoriais entre meio-ambiente, saúde, e economia e os vários pontos de intersecção: viabilidade, equidade, sustentabilidade e governança;

- resultados preliminares e terminais distinguindo-

Tabela - Síntese dos quatro "Estudos Avaliativos" extraídos da literatura contendo a metodologia utilizada, os instrumentos aplicados, os indicadores produzidos, os resultados obtidos e a análise crítica do modelo adotado.

\begin{tabular}{|c|c|c|c|c|c|c|}
\hline Título & Autor & $\begin{array}{l}\text { Metodologia } \\
\text { utilizada }\end{array}$ & Instrumentos aplicados & $\begin{array}{l}\text { Indicadores } \\
\text { produzidos }\end{array}$ & $\begin{array}{l}\text { Sumários dos } \\
\text { principais resultados }\end{array}$ & $\begin{array}{l}\text { Análise crítica do } \\
\text { modelo adotado }\end{array}$ \\
\hline $\begin{array}{l}\text { Healthy Cities } \\
\text { Australia: the } \\
\text { evaluation of the } \\
\text { pilot project in } \\
\text { Noarlunga, } \\
\text { South Australia }\end{array}$ & $\begin{array}{l}\text { Baum \& } \\
\text { Cooke }^{4} \\
\text { (1992) }\end{array}$ & $\begin{array}{l}\text { Metodologia } \\
\text { "construtivista" ao } \\
\text { longo dos três } \\
\text { anos do "piloto" do } \\
\text { projeto, coletando- } \\
\text { se dados através } \\
\text { de questionários, } \\
\text { entrevistas e } \\
\text { surveys, em três } \\
\text { estágios distintos } \\
\text { (1987-1988- } \\
\text { inicial; } 1989 \text { - } \\
\text { intermediário; } \\
\text { 1990 -a conclusão } \\
\text { do "piloto"); } \\
\text { análise da } \\
\text { cobertura da mídia } \\
\text { sobre temas } \\
\text { relacionados com } \\
\text { saúde e ambiente } \\
\text { em três meses } \\
\text { antes de } 1987 \text { e } \\
\text { três meses depois } \\
\text { de } 1988 .\end{array}$ & $\begin{array}{l}\text { Entrevistas com } 34 \\
\text { informantes- chave em } \\
1987-1988 \text { e com } 51 \text { em } \\
\text { 1990; questionários } \\
\text { preenchidos pelos próprios } \\
\text { agentes comunitários de } \\
\text { saúde (22) em 1987-1988 e } \\
\text { com } 37 \text { em } 1990 \text { e com } 23 \\
\text { diretores de escolas } \\
\text { primárias em 1987-1988 e } \\
\text { com } 30 \text { em 1990; survey da } \\
\text { "consciência comunitária" } \\
\text { sobre o projeto com } 307 \\
\text { pessoas em 1989 e com } \\
\text { 319 em 1990; revisão do } \\
\text { gerenciamento e da } \\
\text { referência do comitê através } \\
\text { de questionário. }\end{array}$ & $\begin{array}{l}\text { O s resultados } \\
\text { levaram em conta a } \\
\text { capacidade do projeto } \\
\text { em: (1) formular } \\
\text { políticas públicas } \\
\text { saudáveis, (2) criar } \\
\text { ambientes saudáveis; } \\
\text { (3) fortalecer a ação } \\
\text { comunitária. (4) } \\
\text { desenvolver } \\
\text { habilidades pessoais, } \\
\text { (5) re-orientar os } \\
\text { serviços de saúde na } \\
\text { direção de promoção } \\
\text { da saúde e prevenção } \\
\text { de doenças. }\end{array}$ & $\begin{array}{l}\text { Em relação a formular } \\
\text { políticas públicas } \\
\text { saudáveis e criar } \\
\text { ambientes saudáveis: } \\
\text { (ver artigo para os } \\
\text { outros resultados) } \\
67 \% \text { dos informantes- } \\
\text { chave acharam que o } \\
\text { projeto foi eficaz em } \\
\text { aglutinar planejadores } \\
\text { de diferentes setores } \\
\text { contrastando com } \\
32 \% \text { das diretoras } \\
\text { das escolas } \\
\text { primárias; } 22 \% \text { dos } \\
\text { informantes-chave } \\
\text { acharam que o } \\
\text { projeto foi eficaz em } \\
\text { equacionar as } \\
\text { iniqüidades no } \\
\text { estado de saúde; } \\
\text { grande parte dos } \\
\text { informantes-chave e } \\
\text { dos trabalhadores da } \\
\text { saúde (78\% e } 66 \% \text { ) } \\
\text { acharam que houve } \\
\text { mudanças no que } \\
\text { concerne ao } \\
\text { ambiente, mas a } \\
\text { maior parte das } \\
\text { diretoras de escola e } \\
\text { dos membros da } \\
\text { comunidade ( } 46 \% \text { e } \\
54 \% \text { ) não souberam } \\
\text { informar se houve tais } \\
\text { tipos de mudanças. }\end{array}$ & $\begin{array}{l}\text { A ênfase na } \\
\text { metodologia } \\
\text { qualitativa não } \\
\text { garantiu uma } \\
\text { abordagem } \\
\text { participativa, o que } \\
\text { pode ser assinalada } \\
\text { pelas altas taxas de } \\
\text { "não sabe" obtidas } \\
\text { pelos questionários } \\
\text { e surveys com } \\
\text { diretores de escola } \\
\text { e comunidade. }\end{array}$ \\
\hline $\begin{array}{l}\text { The Valencian } \\
\text { Community } \\
\text { Healthy Cities } \\
\text { Network: } \\
\text { assessment of the } \\
\text { implementation } \\
\text { process }\end{array}$ & $\begin{array}{l}\text { Nunez et } \\
\mathrm{al}^{39} \\
(1994)\end{array}$ & $\begin{array}{l}\text { Aplicação de } \\
\text { questionários } \\
\text { através de } \\
\text { entrevista pessoal } \\
\text { com três } \\
\text { informantes-chave } \\
\text { dos } 48 \text { projetos: } 0 \\
\text { prefeito, membro } \\
\text { do conselho de } \\
\text { saúde e } 0 \\
\text { coordenador do } \\
\text { projeto }\end{array}$ & $\begin{array}{l}\text { Q uestionário com } 57 \\
\text { questões (15 fechadas, } 17 \\
\text { abertas, } 25 \text { semi-abertas) e } \\
\text { medição do nível de } \\
\text { desenvolvimento dos } \\
\text { projetos. }\end{array}$ & $\begin{array}{l}\text { Análise descritiva da } \\
\text { informação usando } \\
\text { D base III e Epiinfo e } \\
\text { score de } 0 \text { a } 2 \text { para } \\
\text { existência ou não de } \\
\text { Diagnóstico de } \\
\text { Saúde, Plano de } \\
\text { Saúde, Comitê } \\
\text { Intersetorial, Canais } \\
\text { de Participação Social }\end{array}$ & $\begin{array}{l}\text { Prefeito participa do } \\
\text { projeto em } 13 / 48 \\
\text { cidades; } 31 / 48 \\
\text { cidades fizeram } \\
\text { diagnóstico de saúde; } \\
11 / 48 \text { cidades } \\
\text { organizaram comitês } \\
\text { intersetoriais; } 18 / 48 \\
\text { cidades } \\
\text { estabeleceram canais } \\
\text { de participação social }\end{array}$ & $\begin{array}{l}\text { Bastante centrado } \\
\text { na organização de } \\
\text { elementos do setor } \\
\text { saúde (Plano de } \\
\text { Saúde, Diagnóstico } \\
\text { de Saúde, } \\
\text { respondentes) e } \\
\text { medidas } \\
\text { exclusivamente de } \\
\text { processo. }\end{array}$ \\
\hline
\end{tabular}


se meios de fins e a relação entre eles, especificando seus respectivos potenciais de promover saúde;

- exames que esclareçam se o movimento de comunidades saudáveis é apenas um exercício de mobilização comunitária ou desenvolvimento de uma estratégia social em saúde, pressupondo-se a análise de aspectos como quem se apropria do projeto, se há controle exclusivo de profissionais, o jogo de poder na definição dos focos de ações, etc.

A Tabela mostra os estudos avaliativos apresentando a metodologia que foi utilizada, os instrumentos aplicados, os indicadores produzidos, resultados obtidos e uma breve análise crítica da metodologia.

Dos quatro "estudos avaliativos" tabulados, três 5,6,39 utilizaram, primordialmente, metodologias qualitativas e buscaram, na percepção dos atores envolvidos, julgamentos quanto à implementação do projeto e quanto aos resultados obtidos preliminarmente. Entretanto, esses atores "participam" apenas como fontes de dados, não se envolvendo efetivamente como sujeitos do processo avaliativo. Como análise dos dados obtidos, há quantificação desta percepção em termos percentuais. Examina-se, também, através de percentuais, a estrutura organizacional dos projetos, em relação à existência ou não de elementos como comitês intersetoriais, diagnósticos e planos municipais de saúde, participação dos prefeitos, mobilização comunitária e outros.

$\mathrm{O}$ artigo de Ouellet, ${ }^{44}$ por outro lado, usa como metodologia uma abordagem holística que leva em conta "modelos de ação negociada" o que proporciona uma avaliação interativa que objetiva envolver os participantes no processo e ajudá-los a obter uma melhor compreensão do projeto. Entretanto, parece que seu objetivo maior não é o desenvolvimento de habilidades avaliativas nos atores locais, mas fazer com que os "avaliadores" também sejam capazes de comunicar, motivar e negociar, e os "avaliados" tenham interesse no processo avaliativo e concordem em ouvir opiniões, que podem ser diferentes das suas, em relação ao desenvolvimento do projeto.

Levando em conta os princípios da WHO, Regional Office for Europe, ${ }^{46,61}$ os quatro estudos adotam múltiplos métodos para coletar dados, mas não pare-

Tabela- Continuacão.

\begin{tabular}{|c|c|c|c|c|c|c|}
\hline Título & Autor & $\begin{array}{l}\text { Metodologia } \\
\text { utilizada }\end{array}$ & Instrumentos aplicados & $\begin{array}{l}\text { Indicadores } \\
\text { produzidos }\end{array}$ & $\begin{array}{l}\text { Sumários dos } \\
\text { principais resultados }\end{array}$ & $\begin{array}{l}\text { Análise crítica do } \\
\text { modelo adotado }\end{array}$ \\
\hline $\begin{array}{l}\text { Preliminary results } \\
\text { of an evaluation } \\
\text { of three Healthy } \\
\text { Cities initiatives } \\
\text { in the Montreal } \\
\text { area }\end{array}$ & $\begin{array}{l}\text { Ouellet } \\
\text { et al }{ }^{44} \\
\text { (1994) }\end{array}$ & $\begin{array}{l}\text { Abordagem } \\
\text { holística, baseada } \\
\text { na teoria do modo } \\
\text { negociado de } \\
\text { ação. A } \\
\text { abordagem } \\
\text { holística coloca as } \\
\text { atividades do } \\
\text { programa no seu } \\
\text { contexto de ação e } \\
\text { examina as } \\
\text { variáveis em um } \\
\text { modelo não linear } \\
\text { de relações. As } \\
\text { fontes dos dados } \\
\text { foram: entrevistas } \\
\text { semi-estruturadas, } \\
\text { análise } \\
\text { documental e } \\
\text { observação de } \\
\text { campo durante as } \\
\text { reuniões dos } \\
\text { comitês. Os dados } \\
\text { foram analisados } \\
\text { de acordo com o } \\
\text { modelo de análise } \\
\text { temática. }\end{array}$ & $\begin{array}{l}\text { Dois tipos de instrumentos: } \\
\text { steering committee (comitê } \\
\text { diretivo) e news-letter". }\end{array}$ & $\begin{array}{l}\text { O ito variáveis foram } \\
\text { estudadas: ambiente } \\
\text { externo, ambiente } \\
\text { interno, coerência dos } \\
\text { objetivos, } \\
\text { participação } \\
\text { dos cidadãos, ação } \\
\text { intersetorial, } \\
\text { estrutura, atividades e } \\
\text { resultados. }\end{array}$ & $\begin{array}{l}\text { O s três projetos } \\
\text { apóiam-se no } \\
\text { processo de } \\
\text { negociação que deve } \\
\text { respeitar a } \\
\text { manutenção do } \\
\text { equilíbrio entre os } \\
\text { objetivos da ação } \\
\text { intersetorial e da } \\
\text { participação dos } \\
\text { cidadãos. }\end{array}$ & $\begin{array}{l}\text { É interessante o fato } \\
\text { de se propor um } \\
\text { modelo de avaliação } \\
\text { no qual participam } \\
\text { os avaliadores } \\
\text { externos e também } \\
\text { os atores envolvidos } \\
\text { no projeto. }\end{array}$ \\
\hline $\begin{array}{l}\text { Healthy Cities } \\
\text { Evaluation: } \\
\text { the co-ordinators } \\
\text { perspective }\end{array}$ & $\begin{array}{l}\text { Booneka } \\
\text { mp et al }\left.\right|^{5} \\
\text { (1999) }\end{array}$ & $\begin{array}{l}\text { Entrevistas semi- } \\
\text { estruturadas com } \\
\text { os coordenadores } \\
\text { dos projetos e } \\
\text { análise } \\
\text { documental. }\end{array}$ & $\begin{array}{l}\text { As entrevistas abordaram: a } \\
\text { concepção de saúde, a } \\
\text { percepção do papel do } \\
\text { governo local na promoção } \\
\text { da saúde, o papel da } \\
\text { comunidade nas políticas } \\
\text { municipais relacionadas } \\
\text { com a saúde, o trabalho } \\
\text { interdepartamental. } \\
\text { Analisou-se a existência de } \\
\text { estratégias para o } \\
\text { desenvolvimento de } \\
\text { políticas públicas saudáveis. } \\
\text { A análise documental } \\
\text { pretendeu identificar e } \\
\text { analisar os setores } \\
\text { envolvidos, a estrutura } \\
\text { organizacional e as relações } \\
\text { formais existentes. }\end{array}$ & $\begin{array}{l}\text { Para cada um dos } \\
\text { temas abordados } \\
\text { pelas entrevistas, } \\
\text { criaram-se categorias } \\
\text { baseadas na "Carta } \\
\text { de Ottawa" e outras } \\
\text { referências. Por } \\
\text { exemplo, determina- } \\
\text { se se o conceito de } \\
\text { saúde emitido pelos } \\
\text { respondentes estava } \\
\text { mais próximo da da } \\
\text { definição da OMS ou } \\
\text { da "Carta de O ttawa" }\end{array}$ & $\begin{array}{l}\text { Considerando a } \\
\text { concepção de saúde, } \\
\text { as ações da } \\
\text { municipalidade para } \\
\text { incrementar a saúde } \\
\text { dos cidadãos, os } \\
\text { objetivos específicos } \\
\text { dos projetos, os } \\
\text { canais de } \\
\text { participação e o } \\
\text { trabalho } \\
\text { interdepartamental. } \\
\text { Essa avaliação } \\
\text { confirmou a natureza } \\
\text { heterogênea dos } \\
\text { projetos de cidades } \\
\text { saudáveis } \\
\text { desenvolvidos. }\end{array}$ & $\begin{array}{l}\text { A presenta as } \\
\text { limitações dos } \\
\text { estudos de caso } \\
\text { que se utilizam de } \\
\text { metodologia e } \\
\text { instrumentos } \\
\text { qualitativos, sem a } \\
\text { possibilidade de } \\
\text { generalização. Por } \\
\text { outro lado, a } \\
\text { realização de } \\
\text { entrevistas com os } \\
\text { coordenadores é } \\
\text { fundamental para a } \\
\text { compreensão dos } \\
\text { projetos e suas } \\
\text { características. }\end{array}$ \\
\hline
\end{tabular}


cem demonstrar que envolvem todos os interessados no processo avaliativo no sentido de construção de uma capacidade crítica que possa desenvolver um modelo próprio de avaliação, delineado localmente.

Para tanto, faz-se necessária a adoção de mecanismos que favoreçam a participação de atores sociais com interesse no processo de avaliação e que facilitem dinâmicas de compartilhamento de histórias, visões de futuro e pactuação em torno de objetivos, metas e indicadores, para que se conforme uma cultura avaliativa baseada no contexto local.

Poland ${ }^{46}$ destaca duas iniciativas concretas de avaliação de comunidades saudáveis, que, segundo ele, se aproximariam mais dos princípios e focos de análise de conteúdos por ele propugnados: the New Mexico Partnership for Healthier Communities e the Canadian Knowledge Development Project. Ambas iniciativas adotam a avaliação, não como o objetivo final, mas como instrumento de construção de capacidades e empowerment de grupos populacionais envolvidos com projetos de comunidades saudáveis.

Tais iniciativas participativas adotam estágios que se complementam e se retroalimentam, simultaneamente. São eles:

- compartilhar uma história comum;

- criar uma visão comum de futuro;

- identificar os diversos atores com interesse no processo avaliativo;

- identificar metas, objetivos e indicadores;

- identificar estratégias para se alcançar metas;

- coletar dados e construir indicadores;

- analisar dados;

- comunicar resultados. $^{58}$

A iniciativa do Novo México, acima referida, é uma metodologia formativa, participativa, combina indicadores qualitativos e quantitativos, é empreendida ao longo do processo de implementação da ação e é desenvolvida por atores internos e externos ao projeto. Com isso, busca-se enfrentar a crítica apontada pela literatura que identificou esta lacuna nos estudos avaliativos publicados, que, tradicionalmente, adotam desenhos somativos, empreendidos por atores externos ao projeto/programa com ênfase em indicadores quantitativos.

\section{CONCLUSÃO}

Muita energia do movimento de "cidades/municí- pios saudáveis" tem sido gasta no desenvolvimento de indicadores quantitativos. ${ }^{46}$ Tais indicadores podem ser úteis como estabelecimento de "medidas de ponto de partida" para definição de prioridades, mas poderia criar falsas expectativas daquilo que poderia ser modificado, no curto e no médio prazo, se for levada em conta apenas a magnitude dos problemas considerados.

Duhl"11 comenta que "infelizmente, mudanças levam tempo para ocorrer. Mudanças na forma de perceber, bem como novas maneiras de entender e de agir, também requerem tempo para se desenvolver. Competências, também, necessitam de tempo para se desenvolver, mudanças no seio das comunidades necessitam do apoio dos vários níveis de governo, do setor privado local e das corporações globais".

Avaliar é gerar conhecimento para sustentar e aprimorar processos ${ }^{11} \mathrm{e}$ isto requer que se engaje o maior número de participantes locais (e não, apenas, pesquisadores) num processo abrangente de reflexão sobre a natureza da organização social e sobre os determinantes de saúde. Sem isso, o movimento de "cidades/municípios saudáveis" estaria imune a ser cooptado por profissionais e habitantes bem intencionados ${ }^{46}$ que poderiam ver o movimento apenas como um exercício para melhorar a provisão dos serviços urbanos e não como uma estratégia de recriar uma nova governança local.

A implementação de um processo de avaliação participativa pode favorecer a aglutinação crítica de atores sociais. A questão, então, é fazer do processo avaliativo, não um exercício meramente científico, que busque possibilidades de se replicar experiências bem sucedidas ou descartar aquelas mal sucedidas, constatando-se "o que é efetivo" ou "o que não é efetivo", mas também procurar se detectar, principalmente, "porque tal projeto é efetivo" ou "porque não é efetivo", numa localidade específica.

Importa, então, desenvolver culturas avaliativas locais que favoreçam o exercício crítico para detectar especificidades e potencializar mudanças de rumo, quando estas forem necessárias. Isto é, o que Fawcet et al $^{12}$ (1996) denominam de iniciativas de comunidades saudáveis por meio de avaliação, como estratégia de produção de conhecimento e amadurecimento de uma auto-análise social. Em outras palavras, avaliar para aprender, agir e mudar. 


\section{REFERÊNCIAS}

1. Akerman M, Massei W, Cabral S, Broch A, Cremomese A, Alves TS et al. A concepção de um projeto de observatório de qualidade de vida: relato de uma experiência realizada em Campinas (SP). Saúde Soc 1997;6:83-99.

2. Almeida ES. Cidade/M unicípio saudável: a questão estratégica, o compromisso político. Saúde Soc 1997;6:71-82.

3. Baum F. Healthy cities and change: social movement or bureacratic tool? Health Prom 1993;8:31-40.

4. Baum F, Cooke R. Healthy Cities Australia: the evaluation of the pilot project in N oarlunga. South Australia Health Prom 1992;7:181-93.

5. Boonekamp GMM M, Colomber C, Tomás A, Nuñez A. Healthy cities evaluation: the co-ordinators perspective. Health Promot Int 1999;14:103-10.

6. Buvinich MR. A importância da avaliação de programas e projetos sociais. Boletim Fundação Banco do Brasil 1999; p. 8.

7. Chuchkova M. The healthy cities project of the world health organization and the approach to its realization. Probl Khig 1994;19:3-9.

8. Costa JLR. Algumas reflexões sobre cidade saudável. Saúde Soc 1997;6:65-70.

9. Dennis LI, Flynn BC, Martin JB. Characteristics of pregnant women, utilization, and satisfaction with prenatal services in St. Petersburg, Russia. Public Health N urs 1995;12:374-7.

10. Duhl L. Guide to preventive services: a commentary. Am J Prev Med 2000;1 Suppl:10-1.

11 Duhl L, Hancock T. Community self-evaluation: a guide to assessing healthy cities. Copenhagen: Healthy Cities Papers/ FAD L; 1999.

12. Fawcet SB, Paine-Andrews A, Francisco V, Schultz J, Richter K, Lewis R. Empowering communities health initiative through evaluation. In: Fetterman D, Kaftarian S, W andersman A, editors. Empowerment evaluation: knowledge and tools for self assessment and accountability. Thousand $O$ aks: Sage Foundation; 1996.

13. Ferraz AS. Pertinência da adoção da filosofia de cidades saudáveis no Brasil. Saúde Debate 1993;41:45-9.

14. Flynn BC. Communicating with the public: community-based nursing research and practice. Public Health N urs 1998;15:165-70.

15. Flynn BC. Partnerships in healthy cities and communities: a social commitment for advanced practice nurses. Adv Pract Nurs Q 1997;2:1-6.

16. Flynn BC. Healthy cities: toward worldwide health promotion. Ann Rev Public Health 1996;17:299-309.
17. Flynn BC, Ray DW, Rider MS. Empowering communities: action research through healthy cities. Health Educ Q 1994;21:395-405.

18. Fryer P. A healthy city strategy three years on: the case of $O$ xford City Council. Health Prom 1988;3:213-17.

19. Garcia Barbero M, Caturla Such J. What are we doing in cardiopulmonary resuscitation training in Europe? An analysis of a survey. Resuscitation 1999;41:225-36.

20. Goumans M. Innovations in a fuzzy domain. Healthy Cities and (health) policy development in the Netherlands and the United Kingdom [Phd Thesis]. Maastricht: Faculty of H ealth Sciences of U niversity of M aastricht; 1997.

21. Goumans M, Springett J. From projects to policy: "Healthy Cities" as a mechanism for policy change for health? Health Prom 1997;2:311-22.

22.Goumans M. What about healthy networks? An analysis of national heal thycities networks in Europe. Health Prom 1992;7:273-81.

23. Hancock T. The evolution, impact and significance of the healthy cities/ healthy communities movement. J Public Health Policy 1993;14:5-18.

24. Harris E, Wills J. Developing healthy local communities at local government level: lessons from the past decade. Aust N Z J Public Health 1997;21(Spec):403-12.

25. Howell F, Doorley P. Employee attitudes to involuntary smoking at the workplace. Dublin Healthy Cities Project. Ir M ed J 1991;84:94-6.

26. International U nion for $\mathrm{H}$ ealth Promotion and Education (IUHPE). The Evidence of $\mathrm{H}$ ealth Promotion Effectiveness. Luxemburgo; 1999.

27. Jewkes R, M urcott A. Community representatives: representing the "community"? Soc Sci M ed 1998;46:843-58.

28. Keinert TMM. Planejamento governamental e políticas públicas: a estratégia "cidades saudáveis". Saúde Soc 1997;6:55-64.

29. Kickbush I. Healthy Cities: a working project and a growing movement. Health Prom 1989;4:77-85.

30. Labonté R. A holosphere of healthy and sustainable communities. Aust J Public Health 1993;17:4-12.

31. Malik AM . Cidades Saudáveis: estratégias em aberto. Saúde Soc 1997;6:19-30.

32. M cIntyre L. The evolution of health promotion. Probe $1992 ; 26: 15-22$.

33. M CQ ueen D. Strenthening the evidence base for health promotion: a report on evidence for the fifth global conference on health promotion. M éxico: WHO ; 2000. 
34. Milewa T, Leeuw de E. Reason and protest in the new urban public health movement: an observation on the sociological analysis of political discourse in the ‘healthy city'. Br J Sociol 1996;47:657-70.

35. Milio N. Healthy Cities: the new public health and supportive research. Health Prom 1990;5:291-97.

36. Mendes EV. U ma agenda para a saúde. São Paulo: HUCITEC; 1996.

37 M endes R. Cidades saudáveis no Brasil e os processos participativos: os casos de Jundiaí e M aceió, agosto 2000 [Tese de D outorado]. São Paulo: Faculdade de Saúde Pública da U niversidade de São Paulo; 2000.

38. Minkler M. Health education, health promotion and the open society: a historical perspective. H ealth Educ Q 1989;16:17-30.

39. Nunes A, Colomer C, Peiro R, Alvarez-Dardet C. The Valencian Community Healthy Cities N etwork: assessment of the implementation process. Health Prom 1994;9:189-98.

40. Nutbean D. Evaluating health promotion: progess, problems and solutions. Health Promot Int 1998;13:27-44.

41. O rganización Panamericana de la Salud (OPS). Propuesta para desarrollar un marco conceptual y operativo para evaluar municipios saludables. Washington (DC); 1999.

42. O rganización Panamericana de la Salud (O PS). Taller de evaluación de municipios saludables: informe final. W ashington (DC); 1999.

43. O ttawa Charter for Health Promotion. Health Prom 1987;1:405-62.

44 O uellet F, D urand D, Forget G. Preliminary results of an evaluation of three healthy cities initiatives in the Montreal area. Health Prom 1994;9:153-9.

45. Phillips DR. U rbanization and human health. Parasitology 1993;106 Suppl: S93-107.

46 Poland BD. Knowledge development and evaluation in, of and for Healthy community initiatives. Part I: guiding principles. Health Prom 1996;11:237-4.

47. Poland BD. Knowledge development and evaluation in, of and for $\mathrm{H}$ ealthy community initiatives. Part II: potential content foci. Health Prom 1996;11:341-9.
48. Ribeiro RC. A construção de um município saudável; descentralização e intersetorialidade: experiência em Fortaleza. Saúde Soc 1997;6:47-54.

49. Robertson A, Minkler M. N ew health promotion movement: a critical examination. Health Educ Q 1994;21:295-312.

50. Rootman I, Goodstadt M, Potvin L, Springett J. H acia una estrutura para la evaluación de la promoción de la salud. Copenhagem: O MS O ficina Regional de Europa; 1997.

51. Rupp G. Toward healthy cities: opportunities for collaboration. J U rban Health 1998;75:401-6.

52 Sabouraud A. A better prospect for city life. W orld Health Forum 1992;13:232-6.

53. Silvestre García A, Colomer Revuelta C, Nolasco Bonmatí A, González Sáez L, Alvarez Dardet Díaz $C$. The income level and life styles: towards a law of inverse prevention? Gac Sanit 1990;4:189-92.

54. Stephenson P, Chalmers B, Kirichenko VF, Regina MA, Wagner $M$. Reducing maternal mortality in St Petersburg. World Health Forum 1997;18:189-93.

55. Tapia Granados JA. Reduction of automobile traffic: urgent health promotion policy. Rev Panam Salud Publica 1998;3:137-51.

56. Tsours AD. The WHO healthy ities project: state of the art and future plans. Health Prom 1995;10:133-41.

57. W allerstein N. Powerlessness, empowerment, and health: implications for health promotion programs. Am J Health Prom 1992;6:197-205.

58. Wallerstein N, Maltrud K, Polacsek M. Participatory evaluation workbook for community initiatives. $\mathrm{N} \mathrm{ew}$ M exico: D epartment of H ealth; 1997.

59. Werna E, Harpham T, Blue I, G oldstein G. H ealthy city projects in developing countries. Londres: Earthscan; 1998.

60. W estphal MF. Municípios saudáveis: aspectos conceituais. Saúde Soc 1997;6:9-18.

61. WHO Regional Office for Europe. Health promotion evaluation: recommendations to policymakers. Copenhagem: WHO Regional Office for Europe; 1998. 